\title{
Magnetic Field-Aligned Electric Currents and Fields in the Magnetosphere and Precipitation of Particles
}

\author{
A. HRušKa \\ Herzberg Institute of Astrophysics, National Research Council of Canada, \\ Ottawa, Canada
}

(Received February 7, 1978)

A collisionless plasma with a non-zero velocity along the fieldlines is considered. A large scale electric field $E_{\|}$due to the differential pitch angle anisotropy between ions and electrons is obtained from the time-independent fluid equations. It is shown that the potential difference corresponding to $E_{\|}$can always be expressed as a function of the magnetic field strength $B$ and of the boundary conditions. A strong electric field $E_{\|}$is generated mainly when the typical random velocities of ions and electrons substantially exceed the systematic velocities of particles across the fieldlines. The pressure term in the equation of motion then may become comparable to the $\boldsymbol{V} \times \boldsymbol{B}$-term and the CGL approach, based on the expansion in the powers of the Larmor radius, is no longer a viable approximation.

Electric current in a static plasma is determined by the equation

$$
\boldsymbol{j}=c\left[\frac{1}{4 \pi} \tau B \lambda-\frac{1}{B}(\nu \cdot \operatorname{grad} \Psi) \boldsymbol{\mu}+\frac{1}{B}(\boldsymbol{\mu} \cdot \operatorname{grad} \Psi+\kappa(\Pi-\Psi)) \nu\right] .
$$

Here $\Pi(\Psi)$ is the parallel (perpendicular) component of the pressure tensor of ions and electrons, $\lambda, \mu$ and $\nu$ are the unit vectors in the directions of the tangents, principal normal and bi-normal to the fieldlines and $\kappa$ and $\tau$ are the curvature and torsion of fieldlines. $\tau$ is defined by

$$
\lambda \cdot \operatorname{grad}\left[\tau\left(1-4 \pi(\Pi-\Psi) / B^{2}\right)\right]=-4 \pi \nu \cdot \operatorname{grad}(\Pi+\Psi) \cdot
$$

Relations (1) and (2) indicate that there are two distinguishable currents in a plasma. One current is flowing perpendicular to $\nu$ and is due to non-zero pressure gradients in this direction while the other current, not explicitly associated with the $\boldsymbol{\nu}$-directed gradients, flows along the $\nu$-lines. In the magnetosphere, we may identify the fieldaligned component of the first current with the Birkeland current and the second current with the ring current. 